Disclosure of Interests: Giulio Olivieri: None declared, Fulvia Ceccarelli: None declared, Alessandra Lo Presti: None declared, silvia angeletti: None declared, Carlo Perricone: None declared, Giancarlo laiani: None declared, Lucia De Florio: None declared, francesca antonelli: None declared, Luigino Amori: None declared, Cristina Garufi: None declared, Francesca Romana Spinelli Grant/ research support from: Pfizer, Speakers bureau: Lilly, BMS, Celgene, cristiano Alessandri: None declared, Guido Valesini: None declared, Massimo Cicozzi: None declared, Fabrizio Conti Speakers bureau: BMS, Lilly, Abbvie, Pfizer, Sanofi DOI: 10.1136/annrheumdis-2020-eular.3592

\begin{tabular}{l|l}
\hline THU0282 & EPIDEMIOLOGY OF CUTANEOUS INVOLVEMENT IN \\
SJÖGREN'S SYNDROME: DATA FROM THREE FRENCH \\
POPULATIONS OF PSS (TEARS, ASSESS, DIAPSS)
\end{tabular}

C. Villon ${ }^{1}$, L. Orgeolet ${ }^{1}$, A. M. Roguedas-Contios ${ }^{1}$, L. Misery ${ }^{1}$, J. E. Gottenberg ${ }^{2}$, D. Cornec ${ }^{1}$, S. Jousse-Joulin ${ }^{1}$, R. Seror ${ }^{3}$, J. M. Berthelot ${ }^{4}$, P. Dieudé ${ }^{3}$, J. J. Dubost ${ }^{5}$, A. L. Fauchais ${ }^{3}$, V. Goeb ${ }^{6}$, E. Hachlla ${ }^{7}$, P. Y. Hatron ${ }^{7}$, C. Larroche ${ }^{3}$, G. Hayem ${ }^{3}$, V. Le Guern ${ }^{3}$, A. Perdriger ${ }^{8}$, J. Morel ${ }^{9}$, O. Vittecoq ${ }^{10}$, X. Mariette ${ }^{3}$, V. Devauchelle-Pensec ${ }^{1}$, A. Saraux ${ }^{1}{ }^{1} \mathrm{CHU}$, Brest, France; ${ }^{2} \mathrm{CHU}$, Strasbourg, France; ${ }^{3} \mathrm{AP}-\mathrm{HP}$, Paris, France; ${ }^{4} \mathrm{CHU}$, Nantes, France; ${ }^{5} \mathrm{CHU}$, Clermont-Ferrand, France; ${ }^{6} \mathrm{CHU}$, Amiens, France; ${ }^{7} \mathrm{CHU}$, Lille, France; ${ }^{8} \mathrm{CHU}$, Rennes, France; ${ }^{9} \mathrm{CHU}$, Montpellier, France; ${ }^{10} \mathrm{CHU}$, Rouen, France

Background: Cutaneous involvement is common during primary Sjogren's Syndrome (pSS) but prevalence and characteristics are difficult to establish precisely because of the limited number of patients studied in most cohorts, the variability of the disorders evaluated in each cohort, the rarity of some of them, and the heterogeneity of evaluations from previous studies (1).

Objectives: To determine the prevalence and significance of dermatological disorders in primary Sjogren Syndrome.

Methods: We use 2 French cohorts (ASSESS, in which prevalence of skin disorders in 395 pSS patients was evaluated, and diapSS in which 91 consecutive pSS patients had an examination by a dermatologist) and baseline data of the TEARS randomized trial (110 patients with recent or active pSS, treated with rituximab or placebo, and evaluated for skin dryness using a visual analogue scale out of 100). Results: Skin manifestations included in the ESSDAI were rare in the ASSESS cohort ( $n=16 / 395,4.1 \%$, mainly purpuras; only 3 had high activity) but associated with activity in the other ESSDAI domains (peripheral neurological $(p<0.001)$, muscular $(p=0.01)$, hematological $(p=0.017)$ and biological $(p=0.017))$, history of arthritis $(p=0.008)$, splenomegaly $(p=0.024)$ and higher gamma globulin level $(p=0.008))$ (Table). Compared to pSS patients not receiving a dermatological consultation, the pSS patients who had a dermatological consultation had significantly more dermatological involvement outside ESSDAI score [42\% (29/69) versus 19.6\% (11/56); $\mathrm{p}=0.008]$. The TEARS study showed a high prevalence of cutaneous dryness (VAS $>50 ; 48.2 \%$ ) and that these dry skin patients had higher pain VAS $(61.5+-$ 28.2 vs $46.8+/-27.0 ; p=0.003)$ and drought $(79.4+/-15.2$ vs $62.5+/-21.7 ; p<0.0001)$.

\begin{tabular}{|c|c|c|c|c|}
\hline & $\begin{array}{l}\text { Cutaneous } \\
\text { involvement }\end{array}$ & $\begin{array}{c}\text { No } \\
\text { Cutaneous } \\
\text { involvement }\end{array}$ & Total & $\begin{array}{c}p \\
\text { values* }\end{array}$ \\
\hline Muscular & 3/16 (18.8) & $10 / 373(2.7)$ & $13 / 389(3.3)$ & 0.001 \\
\hline Peripheral nervous system PNS & $4 / 16(25)$ & $34 / 373(9.1)$ & $38 / 389(9.8) c$ & 0.00001 \\
\hline Biological & $8 / 16(50)$ & $138 / 371(37.2)$ & $\begin{array}{c}146 / 387 \\
(37.7)\end{array}$ & 0.017 \\
\hline Hematologic & $7 / 16(43.8)$ & $55 / 373(14.7)$ & $\begin{array}{c}62 / 389 \\
(15.9)\end{array}$ & 0.017 \\
\hline History of arthritis & $12 / 16(75)$ & $154 / 374(41.2)$ & $\begin{array}{c}166 / 390 \\
(42.6)\end{array}$ & 0.008 \\
\hline History of splenomegaly & $2 / 16(12.5)$ & 10/3762.7) & $12 / 392(3.1)$ & 0.024 \\
\hline History of lymphoma & $0 / 16(0)$ & $18 / 379(4.7)$ & $18 / 395(4.6)$ & 0.372 \\
\hline Mean (SD) ESSDAI score & $14.5(6.8)$ & $4.4(5.1)$ & & 0.00001 \\
\hline $\begin{array}{l}\text { Mean (SD) ESSDAI score after excluding } \\
\text { the points awarded by skin manifestations }\end{array}$ & $8.1(6.2)$ & $4.4(5.1)$ & & 0.014 \\
\hline Gammaglobulin levels (mean +/- SD) & $23.1+/-7.3$ & $18.5+/-8.1$ & - & 0.006 \\
\hline
\end{tabular}

Conclusion: The most common skin disorder is dryness, which is associated with a higher level of pain and overall subjective dryness. ESSDAI skin activity is rare, associated with hypergammaglobulinemia and ESSDAI activity. Systematic dermatological examination is informative for non-specific pSS lesions References:

[1] Orgeolet L, Foulquier N, Misery L, Redou P, Pers J-O, Devauchelle-Pensec $\mathrm{V}$, et al. Can artificial intelligence replace manual search for systematic literature? Review on cutaneous manifestations in primary Sjögren's syndrome. Rheumatol Oxf Engl. 2019 Aug 31;

Disclosure of Interests: Camille Villon: None declared, Laure Orgeolet: None declared, Anne-Marie Roguedas-Contios: None declared, Laurent Misery: None declared, Jacques-Eric Gottenberg Grant/research support from: BMS, Pfizer, Consultant of: BMS, Sanofi-Genzyme, UCB, Speakers bureau: Abbvie, Eli Lilly and Co., Roche, Sanofi-Genzyme, UCB, Divi Cornec: None declared, Sandrine Jousse-Joulin: None declared, Raphaèle Seror Consultant of: BMS UCB Pfizer Roche, Jean-Marie Berthelot: None declared, Philippe Dieudé: None declared Jean-Jacques Dubost: None declared, anne-laure Fauchais: None declared, Vincent Goeb: None declared, Eric Hachlla: None declared, Pierre-Yves Hatron None declared, Claire Larroche: None declared, Gilles Hayem: None declared, Véronique LE GUERN Grant/research support from: UCB for GR2 study (to our institution), Aleth Perdriger: None declared, Jacques Morel: None declared, Olivier VITTECOQ: None declared, Xavier Mariette Consultant of: BMS, Gilead, Medimmune, Novartis, Pfizer, Servier, UCB, Valerie Devauchelle-Pensec: None declared, alain saraux: None declared DOI: 10.1136/annrheumdis-2020-eular. 1847

\section{THU0283 \\ DISTINCT CLINICAL FEATURES OF LATE-ONSET SYSTEMIC LUPUS ERYTHEMATOSUS AMONG MALAYSIAN MULTI-ETHNIC COHORT}

S. S. Shaharir ${ }^{1}$, M. S. Mohamed Said ${ }^{1}$, S. Rajalingham ${ }^{1}$, H. Mahadzir ${ }^{1}$, R. Mustafar ${ }^{1}$, A. Abdul Wahab ${ }^{2} .{ }^{1}$ Universiti Kebangsaan Malaysia Medical Centre, Internal Medicine, Kuala Lumpur, Malaysia; ${ }^{2}$ Universiti Kebangsaan Malaysia Medical Centre, Microbiology and Immunology, Kuala Lumpur, Malaysia

Background: Systemic Lupus Erythematosus (SLE) commonly affects young women in their reproductive age group. However, there is an increase prevalence of late-onset SLE, parallel to the higher life expectancies among general populations worldwide. It has been reported that up to $25 \%$ SLE populations have a later onset of disease and their disease expression and course may be different. Objectives: To determine the clinical features and outcomes of late-onset SLE patients in a multi-ethnic Malaysian cohort.

Methods: Medical records of SLE patients who attended regular follow-up clinics in Universiti Kebangsaan Malaysia Medical Centre (UKMMC) from 2011 until June 2019 were reviewed. Late-onset SLE was defined as the onset of SLE symptoms or diagnosis after the age of 50 years old. Information on their socio-demographics and disease characteristics were obtained from the clinical records. Disease damage was assessed using the SLICC/ACR (Systemic Lupus International Collaborating Clinics/American College of Rheumatology) Damage Index (SDI) scores. The disease characteristics and autoantibody profiles were compared between late-onset and younger onset patients. Damage accrual at disease onset and at 5 years was obtained and compared between the two groups.

Results: A total of 429 patients were included and majority of them were Malays $(n=225,52.4 \%)$ followed by Chinese $(n=180,42)$, Indian $(n=21,4.9 \%)$ and others $(n=3,0.7 \%)$. This multi-ethnic SLE cohort was consisted of predominantyly female patients $(n=372,86.7 \%)$ with disease duration of 9.9 years \pm 6.8 years. A total of $13.8 \%(n=59)$ had late onset SLE with mean onset of disease at $58.1 \pm 6.3$ years while younger group was $27.2 \pm 9.4$ years. The commonest system involvement among the late-onset group was haematological manifestation (69.5\%).Compared to the younger-onset SLE, late-onset SLE occurred significantly higher among the Chinese $(66.1 \%$ ) as compared to Malay (32.3\%), Indians and other ethnics (1.7\%), p<0.01. Patients with late-onset SLE also had significantly less musculoskeletal (37.3\% vs $62.4 \%$ ) and renal $(23.7 \%$ vs $71.1 \%), p<0.001$ and tend to have less muco-cutanoues manifestations ( 28.8 vs $42.4 \%, p=0.06$ ). Meanwhile, pulmonary involvement was more common among the late onset SLE patients (11.9\% vs $0.8 \%, p<0.001)$. Extractable nuclear antigen (ENA) results were available in 197 patients and patients with late-onset SLE had significantly higher rate of anti-RO positive $(63 \%$ vs $3.9 \%), p=0.01$. Otherwise, no significant difference in the other autoantibodies expressions including anti-La, anti-Sm, anti-RNP, anti-ribosomal $P$ and anti-phospholipid antibodies. Patients with late-onset SLE tend to have more damage accrual at 5 years as compared to the younger age group $(p=0.07)$. The mortality in the late onset group was $13.6 \%(n=8)$ as compared to $2.7 \%(n=10)$ in the younger age group, $p=0.01$. Majority of the cause of death in the later onset SLE was infection $(87.5 \%)$ while in the younger age group was infection and active disease (90\%).

Conclusion: Late onset SLE occurs more commonly among Chinese ethnics in Malaysia and Malaysian SLE patients with late onset of the disease have distinct clinical manifestations. Damage accrual at 5 years tend to be higher in the late-onset group and the mortality is significantly higher with the major cause of death is infection. The different disease expression and outcome in late onset SLE suggest different factors in influencing the disease course and hence further studies including their genetic profiles are warranted.

References:

[1] Paula I. Burgos; Graciela S. Alarcón. Late-onset Lupus: Facts and Fiction. Future Rheumatol. 2008;3(4):351-356.

[2] S Stefanidou, C Gerodimos, A Benos et al. Clinical expression and course in patients with late onset systemic lupus erythematosus. Hippokratia. 2013; 17(2): 153-156. 
Acknowledgments: This research was supported by the "Fundamental Research Grant Scheme (FRGS/1/2018/SKK02/UKM/03/1)" by Ministry of Education Malaysia

Disclosure of Interests: Syahrul Sazliyana Shaharir: None declared, Mohd Shahrir Mohamed Said: None declared, Sakthiswary Rajalingham Speakers bureau: Pfizer (500USD), Hazlina Mahadzir: None declared, Ruslinda Mustafar: None declared, Asrul Abdul Wahab: None declared DOI: 10.1136/annrheumdis-2020-eular.272

\section{THU0284 PREVALENCE OF NEUROPSYCHIATRIC LUPUS IN PSYCHOSIS PATIENTS WITH A POSITIVE ANTINUCLEAR ANTIBODY}

M Spies. ${ }^{1,2,3}$, J Gutjahr-Holland. ${ }^{3}$, J. V. Bertouch ${ }^{1,3}$, A Sammel. ${ }^{1,3}$. ${ }^{1}$ Australian Rheumatology Association, Sydney, Australia; ${ }^{2}$ Royal Prince Alfred Hospital, Camperdown, Australia; ${ }^{3}$ Prince Of Wales Hospital, Randwick, Australia

Background: Psychosis is a rare manifestation of Neuropsychiatric Systemic Lupus Erythematosus (NPSLE). Patients with SLE may have Psychosis as part of their initial presentation of disease ${ }^{1}$. Current guidelines do not make a recommendation regarding the use of Antinuclear Antibody (ANA) in the assessment of patients with psychosis ${ }^{2}$. There is limited evidence assessing the utility of ANA testing in this setting.

Objectives: Primary objective: Determine the prevalence of NPSLE in patients admitted to a mental health service with a diagnosis of a psychosis, who have had a positive antinuclear antibody test.

Secondary objectives: Determine the frequency and proportion of positive ANA testing in this patient group. Determine the pattern and titres of positive ANAs. Determine the subsequent investigation, referral and diagnosis of patients with positive ANAs.

Methods: Retrospective chart review of patients admitted to a mental health service of two metropolitan tertiary referral centres, Prince of Wales Hospital (POWH) and Royal Prince Alfred Hospital (RPAH), with a diagnosis of psychosis who had been tested for ANA. Patients were identified using their electronically entered diagnosis based on the International Classification of Disease ${ }^{3}$ codes. Assessment of patient data for SLE used the 2019 ACR/EULAR classification criteria ${ }^{4}$. Decisions regarding attribution of psychosis related events to SLE follows the criteria used by Bortoluzzi et $\mathrm{al}^{5}$.

Results: Between $1^{\text {st }}$ of January 2010 and $31^{\text {st }}$ of March 2018 there were 5585 (POWH) and 4620 (RPAH) mental health admission with an ICD diagnosis of psychosis representing 2451 and 2315 individual patients. 449/2451 (18\%) and $462 / 2315(20 \%)$ patients were tested for ANA. 78/449 (17\%) and 57/462 (12\%) were positive. Discharge data was available for all patients and longterm follow up data was completed for $53 / 78$ (81\% - POWH) patients and 50/57 (88\% - RPAH). The mean follow-up time $43 \pm 23$ months and $51 \pm 29$ months respectively.

At discharge there were four patients who met 2019 ACR/EULAR for SLE. Of these, two patients met criteria for NPSLE. One was diagnosed clinically and treated specifically for NPSLE with intravenous methylprednisolone and rituximab.

There were no additional diagnoses of SLE or NPSLE clinically or by criteria found in the available follow up data. Hence the overall prevalence of NPSLE in patients admitted with psychosis was $1.3 \%, 95 \% \mathrm{Cl}[0,6.9 \%]$ and $1.8 \%, 95 \% \mathrm{Cl}$ [0,9.4\%] respectively.

Conclusion: The prevalence of neuropsychiatric lupus in patients with psychosis and a positive ANA was $1 / 78$ and $1 / 57$ a two tertiary referral centres. This study expands significantly on the limited evidence available as to the expected outcomes of a positive ANA test in a patient with psychosis.

References:

[1] Pego-Reigosa JM, Isenberg DA. Psychosis due to systemic lupus erythematosus: characteristics and long-term outcome of this rare manifestation of the disease. Rheumatology (Oxford). 2008; 47:1498-502.

[2] National Institute for Health and Care Excellence. Psychosis and schizophrenia in adults: prevention and management. London: NICE; 2014. Clinical guideline CG178.

[3] World Health Organization. (2004). ICD-10: international statistical classification of diseases and related health problems: tenth revision, 2nd ed.

[4] Aringer M, et al. 2019 European League Against Rheumatism/American College of Rheumatology classification criteria for systemic lupus erythematosus. Arthritis Rheum. 2019; 71(9): 1400-1412.

[5] Bortoluzzi A, et al. Development and validation of a new algorithm for attribution of neuropsychiatric events in systemic lupus erythematosus. Rheumatology. 2015; 54: 891-898.

Acknowledgments: Gordana Popovic, Statistical Consultant, Stats Central, Mark Wainwright Analytical Centre, University of New South Wales, Sydney, Australia. Disclosure of Interests: None declared

DOI: 10.1136/annrheumdis-2020-eular.1585

\section{THU0285 ANALYSIS OF THE RELATIONSHIP BETWEEN ORGAN DAMAGE AND QUALITY OF LIFE IN PATIENTS WITH SYSTEMIC LUPUS ERYTHEMATOSUS}

Y. Takase ${ }^{1}$, H. Doi ${ }^{1}$, T. Iwasaki ${ }^{1}$, M. Hashimoto ${ }^{2}$, R. Inaba ${ }^{1}$, T. Kozuki ${ }^{1}$,

M. Taniguchi ${ }^{1}$, Y. Tabuchi ${ }^{1}$, K. Kitagori ${ }^{1}$, S. Akizuki ${ }^{1}$, K. Murakami ${ }^{1}$,

R. Nakashima ${ }^{1}$, H. Yoshifuji ${ }^{1}$, W. Yamamoto ${ }^{3}$, M. Tanaka ${ }^{2}$, K. Ohmura ${ }^{1}$.

${ }^{1}$ Graduate School of Medicine, Kyoto University, Department of Rheumatology and Clinical Immunology, Kyoto, Japan; ${ }^{2}$ Graduate School of Medicine, Kyoto

University, Department of Advanced Medicine for Rheumatic Diseases, Kyoto, Japan; ${ }^{3}$ Kurashiki Sweet Hospital, Okayama, Japan

Background: Systemic lupus erythematosus (SLE) is an autoimmune disease that can not only cause systemic symptoms, such as fever and arthritis, but can also damage important organs, such as those of the central nervous system and the kidneys. Prevention of irreversible organ damage is important for bette prognosis [1]. Additionally, the importance of maintaining the quality of life (QOL) of patients has recently been emphasized. However, only a few studies have examined the relationship between irreversible organ damage and patient QOL. Objectives: To assess the relationship between organ damage and QOL, and to survey which organs have more significant effects on QOL.

Methods: We conducted a questionnaire-based survey of 183 patients with SLE at Kyoto University Hospital from September to December 2019. We used the SLICC/ACR Damage Index (SDI) to evaluate organ damage [2]. The following five scales were employed to evaluate QOL: the physical (PCS) and mental component summary (MCS) of the Medical Outcome Study (MOS) 36-Item Short-Form Health Survey version 2.0 (SF-36v2) [3], health (HRQOL) and non-health-related QOL (N-HRQOL) of LupusPRO [4], and SLE Symptom Checklist (SSC) [5].

Results: Linear regression analysis showed significant correlation between the SD score and all QOL scales except for N-HRQOL, suggesting negative effects of organ damage on QOL (Table 1). Next, we analysed whether there was a significant difference in the SF-36 score between those who were positive and negative for each SDI item (41 in total), using the Wilcoxon rank sum test. Muscle atrophy or weakness $\left(p=3.0 \times 10^{-10}\right)$, osteoporosis with fracture or vertebral collapse $\left(p=9.7 \times 10^{-8}\right)$, claudication $\left(p=7.4 \times 10^{-5}\right)$, and cognitive impairment or major psychosis $\left(p=9.9 \times 10^{-5}\right)$ significantly correlated $\left(p<1.2 \times 10^{-3}\right)$ with PCS, and scarring chronic alopecia $(p=$ $3.4 \times 10^{-4}$ ) with MCS (Table 2 ). In addition, the five SDI items significantly correlated with the remaining three QOL scales (HRQOL, N-HRQOL, and SSC; $p<0.05$ ).

Table 1. Relationship between the SDI score and QOL

\begin{tabular}{lllllll}
\hline & \multicolumn{2}{c}{ SF-36 } & & \multicolumn{2}{c}{ LupusPRO } & SSC \\
\cline { 2 - 3 } \cline { 5 - 6 } & PCS & MCS & & HRQOL & N-HRQOL & \\
\hline$p$-value & $<2.0 \times 10^{-16}$ & $1.7 \times 10^{-3}$ & & $2.2 \times 10^{-11}$ & 0.23 & $1.9 \times 10^{-8}$ \\
\hline
\end{tabular}

Table 2. Relationship between each SDI item and the SF-36 score $(p<$ $1.2 \times 10-3)$

\begin{tabular}{lccc}
\hline \multirow{2}{*}{ SDI item } & \multicolumn{2}{c}{ PCS score } & \multirow{2}{*}{$p$-value } \\
\cline { 2 - 3 } & $\begin{array}{c}\text { Positive } \\
\text { (Median (IQR)) }\end{array}$ & $\begin{array}{c}\text { Negative } \\
\text { (Median (IQR)) }\end{array}$ \\
\hline Muscle atrophy/weakness & $33(19-45)$ & $50(43-54)$ & $3.0 \times 10^{-10}$ \\
Osteoporosis with fracture/vertebral collapse & $24(12-32)$ & $49(38-54)$ & $9.7 \times 10^{-8}$ \\
Claudication & $31(19-35)$ & $49(38-54)$ & $7.4 \times 10^{-5}$ \\
Cognitive impairment/psychosis & $27(17-33)$ & $49(38-54)$ & $9.9 \times 10^{-5}$ \\
SDI item & MCS score & $p$-value \\
& Positive & Negative & \\
Scarring chronic alopecia & (Median (IQR)) & (Median (IQR)) & \\
& 42 (29-51) & $49(39-54)$ & $3.4 \times 10^{-4}$
\end{tabular}

Conclusion: We demonstrated that organ damage has negative effects on patient QOL, indicating the importance of preventing irreversible organ damage for maintaining QOL. Moreover, muscle atrophy/weakness, osteoporosis with fracture/vertebral collapse, claudication, cognitive impairment/major psychosis, and scarring chronic alopecia significantly correlated with QOL deterioration, suggesting that these items should be examined with special care in clinical practice. References:

[1] Lopez R, et al. Rheumatology (Oxford). 2012; 51:491-498.

[2] Gladman D, et al. Arthritis Rheum. 1996; 39:363-369.

[3] Fukuhara S, et al. J Clin Epidemiol. 1998; 51:1037-1044

[4] Inoue M, et al. Lupus. 2017; 26:849-856.

[5] Grootscholten C, et al. Qual Life Res. 2003; 12:635-644.

Disclosure of Interests: Yudai Takase: None declared, Hiroshi Doi: None declared, Takeshi Iwasaki: None declared, Motomu Hashimoto Grant/research support from: 